\title{
A MATHEMATICAL MODEL FOR FLUID-GLUCOSE-ALBUMIN TRANSPORT IN PERITONEAL DIALYSIS
}

\author{
ROMAN CHERNIHA *,***, JOANNA STACHOWSKA-PIETKKA**, JACEK WANIEWSKI ** \\ * Institute of Mathematics \\ National Academy of Sciences of Ukraine, Tereshchenkivs'ka Street 3, 01601 Kyiv, Ukraine \\ e-mail: cherniha@gmail.com \\ ** Nałęcz Institute of Biocybernetics and Biomedical Engineering \\ Polish Academy of Sciences, Ks. Trojdena 4, 02-109 Warsaw, Poland \\ e-mail: \{jstachowska, jacekwan\} @ibib.waw.pl \\ ${ }^{* * *}$ School of Mathematical Sciences \\ University of Nottingham, University Park, Nottingham, NG7 2RD, UK \\ e-mail: Roman. Cherniha@nottingham.ac.uk
}

\begin{abstract}
A mathematical model for fluid and solute transport in peritoneal dialysis is constructed. The model is based on a threecomponent nonlinear system of two-dimensional partial differential equations for fluid, glucose and albumin transport with the relevant boundary and initial conditions. Our aim is to model ultrafiltration of water combined with inflow of glucose to the tissue and removal of albumin from the body during dialysis, by finding the spatial distributions of glucose and albumin concentrations as well as hydrostatic pressure. The model is developed in one spatial dimension approximation, and a governing equation for each of the variables is derived from physical principles. Under some assumptions the model can be simplified to obtain exact formulae for spatially non-uniform steady-state solutions. As a result, the exact formulae for fluid fluxes from blood to the tissue and across the tissue are constructed, together with two linear autonomous ODEs for glucose and albumin concentrations in the tissue. The obtained analytical results are checked for their applicability for the description of fluid-glucose-albumin transport during peritoneal dialysis.
\end{abstract}

Keywords: fluid transport, transport in peritoneal dialysis, nonlinear partial differential equation, ordinary differential equation, steady-state solution.

\section{Introduction}

Peritoneal dialysis is a life saving treatment for chronic patients with end-stage renal disease (Gokal and Nolph 1994). The peritoneal cavity, an empty space that separates bowels, abdominal muscles and other organs in the abdominal cavity, is applied as a container for the dialysis fluid, which is infused there through a permanent catheter and left in the cavity for a few hours. During this time small metabolites (urea, creatinine) and other uremic toxins diffuse from blood that perfuses the tissue layers close to the peritoneal cavity to the dialysis fluid, and finally are removed together with the drained fluid. The treatment cycle (infusion, dwell, drainage) is repeated several times every day. The peritoneal transport occurs between the dialysis fluid in the peritoneal cavity and blood passing down the capillaries in the tissue surrounding the peritoneal cavity. The capillaries are distributed within the tissue at different distances from the tissue surface in contact with the dialysis fluid. The solutes, which are transported between blood and the dialysis fluid, have to cross two transport barriers: the capillary wall and a tissue layer (Flessner 2006).

Typically, many solutes are transported from blood to dialysate, but some solutes such as, for example, an osmotic agent (typically glucose), which is present in high concentration in dialysis fluid, are transported in the opposite direction, i.e., to the blood. This kind of transport system appears also in other medical treatments, such as local delivery of anticancer medications, and in some experimental and natural physiological phenomena (see below). Typically a distributed approach is applied to take 
into account spatial properties of these systems. The first applications of the distributed model were limited to the diffusive transport of gases between blood and artificial gas pockets within the body (Piiper et al., 1962), between subcutaneous pockets and blood (Van Liew, 1968; Collins, 1981), and transport of heat and solutes between blood and the tissue (Perl, 1963; 1962).

Applications of the distributed approach to the modeling of diffusive transport of small solutes include the description of transport from the cerebrospinal fluid to the brain (Patlak 1975), delivery of drugs to the human bladder during intravesical chemotherapy, and drug delivery from the skin surface to the dermis in normal and cancer tissue (Gupta et al., 1995; Wientjes et al., 1993; 1991). Finally, the distributed approach was also proposed for a theoretical description of fluid and solute transport in solid tumors (Baxter and Jain, 1989; 1990; 1991). The mathematical descriptions of these systems were obtained using partial differential equations based on the simplification that capillaries are homogeneously distributed within the tissue. Experimental evidence confirmed the good applicability of such models (see, for example, the papers of Waniewski et al. (1996a; 1996b; 2007), Smit et al. (2004a), Flessner (2006), Parikova et al. (2006), Stachowska-Pietka et al. (2012), Guest et al. (2012) and the references therein).

An important objective of peritoneal dialysis is to remove excess water from the patient (Gokal and Nolph, 1994). Typical values of water ultrafiltration measured during peritoneal dialysis are 10-20 $\mathrm{mL} / \mathrm{min}$ (Heimbürger et al., 1992; Waniewski et al., 1996a, 1996b; Smit et al., 2004a; 2004b). This is achieved by inducing osmotic pressure in the dialysis fluid by adding a solute (called the osmotic agent) in high concentration. The most frequently used osmotic agent is glucose. This medical application of high osmotic pressure is unique for peritoneal dialysis. The flow of water from blood across the tissue to the dialysis fluid in the peritoneal cavity carries solutes of different size, including large proteins, and adds a convective component to their diffusive transport.

The mathematical description of fluid and solute transport between blood and dialysis fluid in the peritoneal cavity has not been fully formulated yet, in spite of the well-known basic physical laws for such transport. The complexity of peritoneal fluid transport modelling comes mainly from the fact that, whereas diffusive transport of small solutes is linear, the process of water removal during peritoneal dialysis by osmosis is nonlinear. A first formulation of the general distributed model for combined solute and fluid transport was proposed by Flessner et al. (1984) and applied later for the description of the peritoneal transport of small molecules (Flessner et al., 1985).

The next attempt to model fluid and solute transport did not result in a satisfactory description. It was assumed in that model that the mesothelium is a very efficient osmotic barrier for glucose with the same transport characteristics as the endothelium (Seams et al., 1990). The assumption resulted in negative interstitial hydrostatic pressures during osmotically driven ultrafiltration from blood to the peritoneal cavity during peritoneal dialysis (Seams et al., 1990). This contradicted the experimental evidence on positive interstitial hydrostatic pressure during the ultrafiltration period of peritoneal dialysis (Flessner 1994). Moreover, the mesothelium, being a highly permeable layer, cannot provide enough resistance to small solute transport to be an osmotic barrier for solutes such as glucose (Flessner, 1994; 2006; 2009; Czyzewska et al., 2000).

Recent mathematical, theoretical and numerical studies have introduced new concepts on peritoneal transport and yielded a better description of particular processes such as pure water transport, a combined osmotic fluid flow and small solute transport, and transport of water and proteins (Flessner, 2001; Cherniha and Waniewski, 2005; Stachowska-Pietka et al., 2006; 2007; Cherniha et al., 2007; Waniewski et al., 2007; 2009). A recent study by Stachowska-Pietka et al. (2012) addresses again the combined transport of fluid (water) and several small solutes.

However, the problem of a combined description of osmotic ultrafiltration to the peritoneal cavity, absorption of the osmotic agent from the peritoneal cavity and leak of macromolecules (e.g., albumin) from blood to the peritoneal cavity has not been addressed yet. Therefore, we present here an extended model for these phenomena and investigate its mathematical structure. In particular, we investigate fundamental questions concerning the role of various transport components, such as osmotic and oncotic gradients and the hydrostatic pressure gradient. It should be stressed that the oncotic gradient leading to the leak of macromolecules from blood to the peritoneal cavity has the opposite sign to the osmotic gradient; hence, their combination may lead to new effects, which cannot arise in the case of the simplified models mentioned above. The model addresses also the disputed problem of the values of the Staverman reflection coefficients for glucose and albumin in the tissue, which cannot be directly measured and need to be indirectly derived from clinical data (Katchalsky and Curran, 1965; Waniewski, 2013)

The paper is organized as follows. In Section 2, a mathematical model of glucose and albumin transport in peritoneal dialysis is constructed. In Section 3, non-uniform steady-state solutions of the model are constructed and their properties are investigated. Moreover, these solutions are tested using real parameters that represent clinical treatments of peritoneal dialysis. The results are compared with those derived by numerical simulations for simplified models (Cherniha et al., 
2007; Waniewski et al., 2007). Finally, we present some conclusions and discussion in the last section.

\section{Mathematical model}

Here we present a new model of fluid and solute transport in peritoneal dialysis. The model is developed in one spatial dimension with $x=0$ representing the boundary of the peritoneal cavity and $x=L$ representing the end of the tissue surrounding the peritoneal cavity (see the work of Stachowska-Pietka et al. (2012) for a discussion of the assumptions involved in this approach).

The mathematical description of transport processes within the tissue consists of the local balance of the fluid volume and solute mass. For incompressible fluid, the change of volume may occur only due to elasticity of the tissue. The fractional fluid void volume, i.e., the volume occupied by the fluid in the interstitium (the rest of the tissue being cells and macromolecules forming the solid structure of the interstitium) expressed per unit volume of the whole tissue, is denoted by $\nu(t, x)$, and its time evolution is described as

$$
\frac{\partial \nu}{\partial t}=-\frac{\partial j_{U}}{\partial x}+q_{U}-q_{l}
$$

where $j_{U}(t, x)$ is the volumetric fluid flux across the tissue (ultrafiltration), $q_{U}(t, x)$ is the density of the volumetric fluid flux from blood capillaries to the tissue, and $q_{l}$ is the density of the volumetric fluid flux from the tissue to the lymphatic vessels (hereafter we assume that it is a known positive constant: nevertheless, it can also be a function of hydrostatic pressure (Stachowska-Pietka et al., 2006; 2012).

Similarly to many distributed models, our model involves the spreading of the source within the whole tissue as an approximation to the discrete structure of blood and lymphatic capillaries.

The independent variables are time $t$ and distance $x$ within the tissue from the tissue surface in contact with the dialysis fluid in the direction perpendicular to this surface (flat geometry of the tissue is here assumed with a finite width, see below). The solutes, glucose and albumin, are distributed only within the interstitial fluid (or part of it, see below), and their concentrations in this fluid are denoted by $C_{G}(t, x)$ and $C_{A}(t, x)$, respectively. The equation that describes the local changes of glucose amount in the tissue, $\nu C_{G}$, is

$$
\frac{\partial\left(\nu C_{G}\right)}{\partial t}=-\frac{\partial j_{G}}{\partial x}+q_{G}
$$

where $j_{G}(t, x)$ is the glucose flux through the tissue, and $q_{G}(t, x)$ is the density of the glucose flux from blood. The cellular uptake of absorbed glucose is not taken into account in Eqn. (2) because this process leads to a small correction in bulk absorption of glucose to the capillaries.
So, we neglect these intracellular changes that have been observed experimentally (Zakaria et al., 2000).

Similarly, the equation that describes the local changes of albumin amount in the tissue, $\alpha \nu C_{A}$, is

$$
\frac{\partial\left(\alpha \nu C_{A}\right)}{\partial t}=-\frac{\partial j_{A}}{\partial x}+q_{A}
$$

where $j_{A}(t, x)$ is the albumin flux through the tissue, $q_{A}(t, x)$ is the density of the albumin flux from blood. The coefficient $\alpha<1$ takes into account that only a part of the fractional fluid void volume $\nu$ that is available for fluid is accessible for albumin because of its large molecular size (Flessner, 2001; Stachowska-Pietka et al., 2007). In other words, the inclusion of the term $\alpha \nu$ in (3) implies that $C_{A}(t, x)$ is the concentration of albumin in that part of the interstitium across which the albumin molecules can pass.

In the general case, Eqn. (3) involves a new fluid void volume function $\nu_{A}(t, x)$, which depends on the hydrostatic pressure similarly to the function $\nu$ (see below) and satisfies the inequality $\nu_{A}<\nu$. Hereafter we set $\nu_{A}=\alpha \nu$ for simplicity.

The flows of fluid and solutes through the tissue are described according to linear non-equilibrium thermodynamics. Osmotic pressure of glucose and oncotic pressure of albumin are described by van't Hoff law, i.e., assuming that the corresponding pressures are proportional to the relevant concentrations.

The fluid flux across the tissue is generated by hydrostatic, osmotic, and oncotic (i.e., osmotic pressure of large proteins) pressure gradients according to the extended Darcy law (Katchalsky and Curran 1965):

$$
\begin{aligned}
j_{U}= & -\nu K \frac{\partial P}{\partial x}+\sigma_{T G} \nu K R T \frac{\partial C_{G}}{\partial x} \\
& +\sigma_{T A} \nu K R T \frac{\partial C_{A}}{\partial x}
\end{aligned}
$$

where $K$ is the hydraulic conductivity of the tissue that is assumed constant for simplicity ( $K$ may also depend on the pressure $P$ ), $R$ is the gas constant, $T$ is absolute temperature, and $\sigma_{T G}$ and $\sigma_{T A}$ are the Staverman reflection coefficients for glucose and albumin in the tissue, respectively. The Staverman reflection coefficient $\sigma$ is a thermodynamic parameter and describes the effectiveness of osmotic pressure in selectively permeable membrane: if $\sigma=0$, then no osmotic pressure can be induced by this solute across the membrane, and if $\sigma=$ 1 , the maximal theoretically possible osmotic effect is induced (ideal semi-permeable membrane).

The fluid flows along the hydrostatic pressure gradient (i.e., from higher to lower pressure) and up the concentration gradient of the osmotic agent (i.e., from solution of lower concentration to solution of higher concentration, osmosis), and therefore there is a difference in signs at the gradients in Eqn. (4). Intermediate values of $\sigma$ represent non-ideal semipermeable membranes. 
Katchalsky and Curran (1965) thoroughly address the problem of Staverman reflection coefficients.

The density of the fluid flux from blood to the tissue is generated, according to the Starling law, by the hydrostatic, osmotic and oncotic pressure differences between blood and the tissue:

$$
\begin{aligned}
q_{U}= & L_{p} a\left(P_{B}-P\right)-L_{p} a \sigma_{G} R T\left(C_{G B}-C_{G}\right) \\
& -L_{p} a \sigma_{A} R T\left(C_{A B}-C_{A}\right),
\end{aligned}
$$

where $P(t, x)$ is the hydrostatic pressure, $L_{p} a$ is the hydraulic conductance of the capillary wall, $P_{B}$ is the hydrostatic pressure of blood, $C_{G B}$ and $C_{A B}$ are the glucose and albumin concentrations in blood, and $\sigma_{G}$ and $\sigma_{A}$ are Staverman reflection coefficients for glucose and albumin in the capillary wall, respectively. Contrary to other parameters, there is an unsolved problem of the values of $\sigma_{G}$ and $\sigma_{A}$. In particular, the values of $\sigma_{G}$ were found low (about $0.005-0.03$ ) in many experiments in contrast to some newer experimental data that suggest the values close to 0.5 (see the discussion of this controversy in the works of Waniewski et al. (2009) and Stachowska-Pietka et al. (2012)). We also assume that blood concentrations of glucose and albumin are constant according to clinical and experimental data that demonstrate only negligible variation of these concentrations during peritoneal dwell of dialysis fluid (Heimburger et al. 1992). This observation is related to the quasi-continuous mode of continuous ambulatory peritoneal dialysis with fluid exchanges every few hours and was applied in most previous theoretical and numerical studies on peritoneal dialysis.

The glucose flux across the tissue is composed of a diffusive component (proportional to the glucose concentration gradient) and a convective component (proportional to glucose concentration and the fluid flux)

$$
j_{G}=-\nu D_{G} \frac{\partial C_{G}}{\partial x}+S_{T G} C_{G} j_{U},
$$

where $D_{G}$ is the diffusivity of glucose in tissue, $S_{T G}$ is the sieving coefficients of glucose in the tissue. According to non-equilibrium thermodynamics, $S_{T G}=1-\sigma_{T G}$ for a homogenous membrane (Katchalsky and Curran 1965).

The density of the glucose flux between blood and tissue describes the number of moles of glucose per unit total volume of tissue per unit time that move between blood and the tissue. It has a diffusive component (proportional to the difference of glucose concentration in blood, $C_{G B}$, and glucose concentration in the tissue, $C_{G}$ ), a convective component (proportional to the density of the fluid flow from blood to the tissue, $q_{U}$ ) and a component that represents lymphatic absorption of solutes (proportional to the density of the volumetric lymph flux, $\left.q_{l}\right)$ :

$$
q_{G}=p_{G} a\left(C_{G B}-C_{G}\right)+S_{G} q_{U} C_{G}-q_{l} C_{G} .
$$

where $p_{G} a$ is the diffusive permeability of the capillary wall for glucose.

In a similar way, the albumin flux across the tissue, $j_{A}(t, x)$, and the density of the albumin flux to the tissue, $q_{A}(t, x)$, can be described as

$$
\begin{aligned}
& j_{A}=-\alpha \nu D_{A} \frac{\partial C_{A}}{\partial x}+S_{T A} C_{A} j_{U}, \\
& q_{A}=p_{A} a\left(C_{A B}-C_{A}\right)+S_{A} q_{U} C_{A}-q_{l} C_{A},
\end{aligned}
$$

where $S_{T A}=1-\sigma_{T A}$ is the sieving coefficient of albumin in the tissue, $S_{A}=1-\sigma_{A}$ is the sieving coefficient of glucose and albumin in the capillary wall, $D_{A}$ is the diffusivity of albumin in the tissue, and $p_{A} a$ is the diffusive permeability of the capillary wall for albumin. Typical values of the model parameters are listed in Table 1.

Equations (1)-(3), together with Eqns. (4)-(9) for flows, form a system of three nonlinear partial differential equations with four variables: $\nu, P, C_{A}$, and $C_{G}$. Therefore, an additional, constitutive, equation is necessary, and this is the equation describing how fractional fluid void volume, $\nu$, depends on interstitial pressure, $P$. This dependence can be established using data from experimental studies (Stachowska-Pietka et al., 2006). It turns out that

$$
\nu=F(P),
$$

where $F$ is a monotonically non-decreasing bounded function with the limits $F \rightarrow \nu_{\min }$ if $P \rightarrow P_{\min }$ and $F \rightarrow \nu_{\max }$ if $P \rightarrow P_{\max }$ (particularly, one may take $\left.P_{\min }=-\infty, P_{\max }=\infty\right)$. Here $\nu_{\min }<1$ and $\nu_{\max }<1$ are empirically measured constants. An example of the function $F$ based on experimental data taken from the work of Zakaria et al. (1999) was presented by Stachowska-Pietka et al. (2006) and Cherniha et al. (2007).

Boundary conditions for a tissue layer of width $L$ impermeable at $x=L$ and in contact with the dialysis fluid at $x=0$ are

$$
\begin{aligned}
x=0: P=P_{D}, \quad C_{G}=C_{G D}, \quad C_{A}=C_{A D}, \\
e x=L: \quad \frac{\partial P}{\partial x}=0, \quad \frac{\partial C_{G}}{\partial x}=0, \quad \frac{\partial C_{A}}{\partial x}=0 .
\end{aligned}
$$

Generally speaking, intraperitoneal pressure $P_{D}$, glucose $C_{G D}$ and albumin $C_{A D}$ concentrations in the peritoneal cavity may depend on time. However, experimental data and theoretical studies suggest that they change at a low rate compared to the rate of transport processes in the tissue (Stachowska-Pietka et al., 2006; 2007; Waniewski, 2007). Therefore, we may assume that $P_{D}, C_{G D}$ and $C_{A D}$ are constant for some time period and assess the steady-state solution for these particular boundary conditions that may be considered an approximated quasi 
steady-state solution for the full model of peritoneal dialysis with time-dependent boundary conditions. This approximation was applied to previous models with variable boundary conditions for small solutes (as glucose) and water transport (but without proteins, as albumin) (see Dedrick, 1981; Flessner, et al., 1984; 1985; Seames et al., 1990; Stachowska-Pietka et al., 2006; 2007; Waniewski, 2001; 2002; Waniewski et al., 2009).

The initial conditions describe an equilibrium within the tissue without any contact with the dialysis fluid:

$$
t=0: P=P^{*}, \quad C_{G}=C_{G}^{*}, \quad C_{A}=C_{A}^{*},
$$

where $P^{*}, C_{G}^{*}$, and $C_{A}^{*}$ are some non-negative values, which will be estimated below.

Note that Eqns. (1)-(10) can be united into three nonlinear partial differential equations (PDEs) for hydrostatic pressure $P(t, x)$, glucose concentration $C_{G}(t, x)$ and albumin concentration $C_{A}(t, x)$. Thus, these three PDEs, together with the boundary and initial conditions 111-13), form a nonlinear boundary-value problem. Possible values of the parameters arising in this problem are presented in Table 1 (see the relevant comments in Section 4).

The fluid flux $j_{U}(t, x)$ at $x=0$ describes the net ultrafiltration flow, i.e., the exchange of fluid between the tissue and the peritoneal cavity across the peritoneal surface, and therefore directly the efficiency of water removal during peritoneal dialysis. The assessment of ultrafiltration flow is important from a practical point of view because low values of this flow in some patients indicate that some problems with osmotic fluid removal have occurred, which may finally result in a failure of the therapy (Parikova, 2006).

\section{Steady-state solutions of the model and their applications}

The time needed to approach the steady state is of the order of minutes for small solutes, as glucose, but increases for larger solutes, and for albumin may be expected to be a few hours (Waniewski, 2007; 2008; Stachowska-Pietka et al., 2006). Thus, the transport of small solutes is close to the steady state even during a single exchange of the dialysis fluid. We should, however, take also into account that patients are in continuously repeated treatment with several hours of fluid dwell in the peritoneal cavity and 10-20 minutes of fluid exchange, and there are typically four exchanges every day (Stachowska-Pietka et al., 2006). Thus, the transport system for large molecules after many exchanges is also close to the steady state. Hence, solutions for the steady state of the system may be considered good approximations for real conditions in the tissue in this clinical setting.
First of all, we consider the special case of tissue in its physiological state without dialysis, and, therefore, no transport to the peritoneal cavity occurs. In this case the boundary conditions at $x=0$ given by Eqn. (11) are replaced by zero Neumann conditions, and the steady-state solution can be easily found because it does not depend on $x$. In fact, by solving the algebraic equations

$$
q_{U}-q_{l}=0, \quad q_{G}=0, \quad q_{A}=0,
$$

one easily obtains the spatially uniform steady-state solution

$$
\begin{aligned}
C_{G}^{*}= & \frac{p_{G} a}{p_{G} a+\sigma_{G} q_{l}} C_{G B}, \\
C_{A}^{*}= & \frac{p_{A} a}{p_{A} a+\sigma_{A} q_{l}} C_{A B}, \\
P^{*}= & P_{B}-\frac{q_{l}}{L_{p} a} \\
& -q_{l} R T\left(\frac{\sigma_{G}^{2} C_{G B}}{p_{G} a+\sigma_{G} q_{l}}+\frac{\sigma_{A}^{2} C_{A B}}{p_{A} a+\sigma_{A} q_{l}}\right) .
\end{aligned}
$$

In the case $q_{l}=0$, i.e., the zero flux from the tissue to the lymphatic vessels, the formulae (15) produce

$$
C_{G}^{*}=C_{G B}, \quad C_{A}^{*}=C_{A B}, \quad P^{*}=P_{B},
$$

otherwise

$$
C_{G}^{*}<C_{G B}, \quad C_{A}^{*}<C_{A B}, \quad P^{*}<P_{B} .
$$

This uniform solution describes the system in equilibrium if no dialysis is performed, and therefore we may use the values $P^{*}, C_{G}^{*}$, and $C_{A}^{*}$ calculated above as the initial profile for simulation of the transport processes after the initiation of dialysis (see the formulae (13)).

To find spatially non-uniform steady-state solutions, we reduce Eqns. (1)- (3) to an equivalent form by introducing scaled non-dimensional independent and dependent variables (except for $\nu$, which is a non-dimensional variable),

$$
\begin{gathered}
x^{*}=\frac{x}{L}, \quad t^{*}=\frac{K P_{D} t}{L^{2}}, \\
p\left(t^{*}, x^{*}\right)=\frac{P}{P_{D}}, \\
u\left(t^{*}, x^{*}\right)=\frac{C_{G}-C_{G B}}{C_{G D}-G_{G B}}, \\
w\left(t^{*}, x^{*}\right)=\frac{C_{A}}{C_{G D}-G_{G B}} .
\end{gathered}
$$

Here we consider only the case with $C_{G D}>C_{G B}$, and therefore variables $u$ and $w$ are well defined and positive, if the appropriate initial conditions, Eqns. (13), (16) and (17), are assumed. For alternative methods of scaling, see the works of Waniewski $(2001 ; 2002)$. 
Thus, taking into account Eqns. (4), (6), and (8), and after rather simple calculations, one obtains Eqns. (1)-(3) in the form (hereafter the upper index $*$ is omitted)

$$
\begin{gathered}
\frac{\partial \nu}{\partial t}=\frac{\partial}{\partial x}\left(\frac{\nu \partial p}{\partial x}\right)-t_{0} \sigma_{1} \frac{\partial}{\partial x}\left(\frac{\nu \partial u}{\partial x}\right) \\
-t_{0} \sigma_{2} \frac{\partial}{\partial x}\left(\frac{\nu \partial w}{\partial x}\right)+t_{0}\left(q_{U}-q_{l}\right), \\
\frac{\partial}{\partial t}\left(\nu\left(u+u_{0}\right)\right) \\
=d_{1} t_{0} \frac{\partial}{\partial x}\left(\frac{\nu \partial u}{\partial x}\right)+S_{T G} \frac{\partial}{\partial x}\left(\frac{u \nu \partial p}{\partial x}\right) \\
-S_{T G} t_{0} \sigma_{1} \frac{\partial}{\partial x}\left(\frac{u \nu \partial u}{\partial x}\right)-S_{T G} t_{0} \sigma_{2} \frac{\partial}{\partial x}\left(\frac{u \nu \partial w}{\partial x}\right) \\
-S_{T G} u_{0} \frac{\partial}{\partial x}\left(\frac{\nu \partial p}{\partial x}\right)-S_{T G} u_{0} t_{0} \sigma_{1} \frac{\partial}{\partial x}\left(\frac{\nu \partial u}{\partial x}\right) \\
+S_{T G} u_{0} t_{0} \sigma_{2} \frac{\partial}{\partial x}\left(\frac{\nu \partial w}{\partial x}\right) \\
+t_{0} S_{G}\left(u+u_{0}\right) q_{U}-t_{0} b_{1} u-t_{0} u_{0} q_{l},
\end{gathered}
$$

$$
\begin{aligned}
& \frac{\partial(\alpha \nu w)}{\partial t} \\
& =d_{2} t_{0} \frac{\partial}{\partial x}\left(\frac{\nu \partial w}{\partial x}\right)+S_{T A} \frac{\partial}{\partial x}\left(\frac{w \nu \partial p}{\partial x}\right) \\
& \quad-S_{T A} t_{0} \sigma_{1} \frac{\partial}{\partial x}\left(\frac{w \nu \partial u}{\partial x}\right)-S_{T A} t_{0} \sigma_{2} \frac{\partial}{\partial x}\left(\frac{w \nu \partial w}{\partial x}\right) \\
& \quad+t_{0} S_{A} w q_{U}-t_{0} b_{2} w-t_{0} w_{0}\left(b_{2}-q_{l}\right),
\end{aligned}
$$

where

$$
\begin{aligned}
q_{U} & =\beta\left(\frac{1}{t_{0}}\left(p_{0}-p\right)+\frac{\sigma_{G} \sigma_{1}}{\sigma_{T G}} u+\frac{\sigma_{A} \sigma_{2}}{\sigma_{T A}} w^{*}\right), \\
\sigma_{1} & =\sigma_{T G} K R T \frac{C_{G D}-G_{G B}}{L^{2}}, \quad d_{1}=\frac{D_{G}}{L^{2}}, \\
\sigma_{2} & =\sigma_{T A} K R T \frac{C_{G D}-G_{G B}}{L^{2}}, \quad d_{2}=\frac{\alpha D_{A}}{L^{2}}, \\
b_{1} & =p_{G} a+q_{l}, \quad b_{2}=p_{A} a+q_{l}, \quad \beta=\frac{L_{p} a L^{2}}{K}, \\
u_{0} & =\frac{C_{G B}}{C_{G D}-G_{G B}}, \quad w_{0}=\frac{C_{A B}}{C_{G D}-G_{G B}}, \\
p_{0} & =\frac{P_{B}}{P_{D}}, \quad t_{0}=\frac{L^{2}}{K P_{D}} .
\end{aligned}
$$

Since Eqns. (21)- 22) have essentially a different structure, we modify them using Eqn. (20) and the notation $w^{*}=w-w_{0}$; hence, the system of governing equations consisting of Eqn. (20) and the equations

$$
\begin{aligned}
& \frac{\partial(\nu u)}{\partial t}+\sigma_{T G} u_{0} \frac{\partial \nu}{\partial t} \\
& =d_{1} t_{0} \frac{\partial}{\partial x}\left(\frac{\nu \partial u}{\partial x}\right)+S_{T G} \frac{\partial}{\partial x}\left(\frac{u \nu \partial p}{\partial x}\right) \\
& \quad-S_{T G} t_{0} \sigma_{1} \frac{\partial}{\partial x}\left(\frac{u \nu \partial u}{\partial x}\right)-S_{T G} t_{0} \sigma_{2} \frac{\partial}{\partial x}\left(\frac{u \nu \partial w}{\partial x}\right) \\
& \quad+t_{0}\left(S_{G} u+u_{0}\left(S_{G}-S_{T G}\right)\right) q_{U}-t_{0} b_{1} u \\
& \quad-t_{0} \sigma_{T G} u_{0} q_{l}
\end{aligned}
$$

$$
\begin{aligned}
& \frac{\partial(\alpha \nu w)}{\partial t}-S_{T A} w_{0} \frac{\partial \nu}{\partial t} \\
& =d_{2} t_{0} \frac{\partial}{\partial x}\left(\frac{\nu \partial w}{\partial x}\right)+S_{T A} \frac{\partial}{\partial x}\left(\frac{w^{*} \nu \partial p}{\partial x}\right) \\
& \quad-S_{T A} t_{0} \sigma_{1} \frac{\partial}{\partial x}\left(\frac{w^{*} \nu \partial u}{\partial x}\right)-S_{T A} t_{0} \sigma_{2} \frac{\partial}{\partial x}\left(\frac{w^{*} \nu \partial w}{\partial x}\right) \\
& \left.\quad+t_{0}\left(S_{A} w-S_{T A} w_{0}\right)\right) q_{U}-t_{0} b_{2} w^{*}-t_{0} \sigma_{T A} w_{0} q_{l}
\end{aligned}
$$

is obtained.

We want to find the steady-state solutions of Eqns. (20), 240-(25) satisfying the boundary conditions (11)-(12). They take the form

$$
\begin{aligned}
& x=0: p=1, \quad u=1, \quad w=\frac{C_{A D}}{C_{G D}-G_{G B}}, \\
& x=1: \frac{\partial p}{\partial x}=0, \quad \frac{\partial u}{\partial x}=0, \quad \frac{\partial w}{\partial x}=0 .
\end{aligned}
$$

for the non-dimensional variables.

Note that, to find the steady-state solutions, Eqns. 201, 24 - 25) can be reduced to the system of ordinary differential equations (ODEs)

$$
\begin{gathered}
\frac{1}{t_{0}} \frac{\mathrm{d}}{d x}\left(\frac{\nu d p}{\mathrm{~d} x}\right)-\sigma_{1} \frac{\mathrm{d}}{\mathrm{d} x}\left(\frac{\nu \mathrm{d} u}{\mathrm{~d} x}\right)-\sigma_{2} \frac{\mathrm{d}}{\mathrm{d} x}\left(\frac{\nu \mathrm{d} w}{\mathrm{~d} x}\right) \\
=q_{l}-q_{U}, \\
d_{1} \frac{\mathrm{d}}{\mathrm{d} x}\left(\frac{\nu \mathrm{d} u}{\mathrm{~d} x}\right)+\frac{S_{T G}}{t_{0}} \frac{\mathrm{d}}{\mathrm{d} x}\left(\frac{u \nu \mathrm{d} p}{\mathrm{~d} x}\right) \\
-S_{T G} \sigma_{1} \frac{\mathrm{d}}{\mathrm{d} x}\left(\frac{u \nu \mathrm{d} u}{\mathrm{~d} x}\right)-S_{T G} \sigma_{2} \frac{\mathrm{d}}{\mathrm{d} x}\left(\frac{u \nu \mathrm{d} w}{\mathrm{~d} x}\right) \\
+\left(S_{G} u+u_{0}\left(S_{G}-S_{T G}\right)\right) q_{U}-b_{1} u-\sigma_{T G} u_{0} q_{l}=0, \\
d_{2} \frac{\mathrm{d}}{\mathrm{d} x}\left(\frac{\nu \mathrm{d} w}{\mathrm{~d} x}\right)+\frac{S_{T A}}{t_{0}} \frac{\mathrm{d}}{\mathrm{d} x}\left(\frac{w^{*} \nu \mathrm{d} p}{\mathrm{~d} x}\right) \\
-S_{T A} \sigma_{1} \frac{\mathrm{d}}{\mathrm{d} x}\left(\frac{w^{*} \nu \mathrm{d} u}{\mathrm{~d} x}\right)-S_{T A} \sigma_{2} \frac{\mathrm{d}}{\mathrm{d} x}\left(\frac{w^{*} \nu \mathrm{d} w}{\mathrm{~d} x}\right) \\
+\left(S_{A} w-S_{T A} w_{0}\right) q_{U}-b_{2} w^{*}-\sigma_{T A} w_{0} q_{l}=0 .
\end{gathered}
$$


The non-linear system of ODEs (28)-30 is still very complex and cannot be integrated in the case of arbitrary coefficients. Thus, we look for correctly specified coefficients, for which this system can be simplified. It can be noted that the relations

$$
S_{A}=S_{T A}, \quad S_{G}=S_{T G}
$$

lead to an essential (this means that automatically $\sigma_{G}=$ $\left.\sigma_{T G}, \sigma_{A}=\sigma_{T A}\right)$ simplification of this system. This assumption is introduced for mathematical reasons only: a specific symmetry of the equations allows much easier rigorous analysis. On the other hand, it is shown in the next section that even in this special case the solutions of the model are qualitatively/quantitatively similar to those obtained via other simplified models, which do not use this assumption.

So, using the assumption (31), expressions for $q_{U}$ from (23) and $j_{U}$ from (4), rewritten in non-dimensional variables

$$
j_{U}=L \nu\left(-\frac{1}{t_{0}} \frac{\partial p}{\partial x}+\sigma_{1} \frac{\partial u}{\partial x}+\sigma_{2} \frac{\partial w}{\partial x}\right),
$$

we obtain the relation

$$
j_{U}=\frac{K \nu}{L_{p} a L} \frac{d q_{U}}{d x}
$$

allowing $j_{U}$ to be found provided the function $q_{U}$ is known. Using the formulae 31-33), the nonlinear ODE system (28)- (30) can be simplified to the form

$$
\begin{gathered}
\frac{1}{\beta} \frac{d}{d x}\left(\nu \frac{d q_{U}}{d x}\right)-q_{U}+q_{l}=0, \\
d_{1} \frac{\mathrm{d}}{\mathrm{d} x}\left(\nu \frac{\mathrm{d} u}{\mathrm{~d} x}\right)-\frac{S_{G}}{\beta} \frac{\mathrm{d}}{\mathrm{d} x}\left(\nu u \frac{\mathrm{d} q_{U}}{\mathrm{~d} x}\right) \\
+S_{G} u q_{U}-b_{1} u-\sigma_{G} u_{0} q_{l}=0, \\
d_{2} \frac{\mathrm{d}}{\mathrm{d} x}\left(\nu \frac{\mathrm{d} w}{\mathrm{~d} x}\right)-\frac{S_{A}}{\beta} \frac{\mathrm{d}}{\mathrm{d} x}\left(\nu w \frac{d q_{U}}{d x}\right) \\
+S_{A} w q_{U}-b_{2}\left(w-w_{0}\right)-w_{0} q_{l}=0 .
\end{gathered}
$$

The linear semi-coupled system of ODEs (33) -34 can be extracted to find the functions $q_{U}$ and $j_{U}$ provided the function $\nu$ is known. However, $\nu$ depends on pressure, which is also an unknown function, and therefore we need to assume additional restrictions on the function $F$ from the formula 10 .

Let us consider the first case, in which we assume that $F$ is a constant function. This assumption was applied in many studies, especially for the description of small solute transport (Dedrick, 1981; Flessner, 1984; Waniewski, 2001; 2002). In this case,

$$
\nu(x)=\nu_{m},
$$

where $\nu_{m}$ is a positive constant. Substituting 37) into the system 33- 34, its general solution can be found:

$$
\begin{gathered}
q_{U}=C_{1} e^{-\lambda x}+C_{2} e^{\lambda x}+q_{l}, \\
j_{U}=\frac{L}{\lambda}\left(-C_{1} e^{-\lambda x}+C_{2} e^{\lambda x}\right), \quad \lambda=\sqrt{\frac{L_{p} a L^{2}}{K \nu_{m}}} .
\end{gathered}
$$

The arbitrary constants $C_{1}$ and $C_{2}$ can be specified using the boundary conditions (26)-27) since the functions $q_{U}$ and $j_{U}$ are expressed via $p, u, w$ and its first-order derivatives (see the formulae (23) and (32)). Making rather simple calculations, we obtain

$$
C_{1}=\left(q_{0}-q_{l}\right) \frac{e^{2 \lambda}}{1+e^{2 \lambda}}, \quad C_{2}=\left(q_{0}-q_{l}\right) \frac{1}{1+e^{2 \lambda}},
$$

where

$$
q_{0}=\beta\left(\frac{1}{t_{0}}\left(p_{0}-1\right)+\sigma_{1}+\sigma_{2} \frac{C_{A D}-C_{A B}}{C_{G D}-G_{G B}}\right) .
$$

Having the explicit formulae for $q_{U}$ and $j_{U}$, Eqns. (35) and (36) can be reduced to two linear autonomous ODEs:

$$
d_{1} \nu_{m} \frac{\mathrm{d}^{2} u}{\mathrm{~d} x^{2}}+\frac{S_{G}}{\lambda}\left(C_{1} e^{-\lambda x}-C_{2} e^{\lambda x}\right) \frac{\mathrm{d} u}{\mathrm{~d} x}-\kappa_{1} u=u_{01}
$$

and

$$
\begin{aligned}
d_{2} \nu_{m} \frac{\mathrm{d}^{2} w}{\mathrm{~d} x^{2}}+\frac{S_{A}}{\lambda}\left(C_{1} e^{-\lambda x}\right. & \left.-C_{2} e^{\lambda x}\right) \frac{\mathrm{d} w}{\mathrm{~d} x} \\
& -\kappa_{2}\left(w-w_{0}\right)=w_{01}
\end{aligned}
$$

with unknown functions $u(x)$ and $w(x)$. Hereafter the notation

$$
\begin{array}{ll}
\kappa_{1}=p_{G} a+\sigma_{G} q_{l}, & u_{01}=\sigma_{G} u_{0} q_{l}, \\
\kappa_{2}=p_{A} a+\sigma_{A} q_{l}, & w_{01}=\sigma_{A} w_{0} q_{l}
\end{array}
$$

is used. Note the similarities in the structure of Eqns. (42) and (43) However, to the best of our knowledge, the general solutions of the ODEs (42) and (43) are unknown. On the other hand, since the unknown functions $u(x)$ and $w(x)$ should satisfy the boundary conditions 26-27, the corresponding linear problems can be numerically solved using, e.g., the Maple program package. Finally, using two expressions for $q_{U}$ from (23) and (38), we obtain the function

$$
\begin{aligned}
p(x)= & p_{0}+t_{0} \sigma_{1} u+t_{0} \sigma_{2}\left(w-w_{0}\right) \\
& -\frac{t_{0}}{\beta}\left(C_{1} e^{-\lambda x}+C_{2} e^{\lambda x}+q_{l}\right) .
\end{aligned}
$$

In the next section, the numerical non-uniform steady-state solutions based on realistic parameter values arising in the formulae derived above will be presented for this case, i.e., with the restrictions (31) and 37). 
Let us now consider the second type of restriction on the function $\nu$. Instead of the rather restrictive assumption (37), we examine the case when the function $\nu$ is non-constant and satisfies the general conditions described after the formula (10). According to the experimental data, the hydrostatic pressure during peritoneal dialysis is a decreasing function with respect to the distance $x$ from the peritoneal cavity (Flessner, 1994; Zakaria et al. 1999; 2000). Hence, function $F(p(x))$ is decreasing (with respect to $x$ ) provided $p(x)$ is a spatially non-uniform steady-state solution. The simplest case of such a pattern occurs when $\nu$ is the linear, monotonically decreasing function of $x$ :

$$
\nu(p(x)) \equiv \nu(x)=\nu_{\max }-\left(\nu_{\max }-\nu_{\min }\right) x
$$

where $x \in[0,1]$. Substituting (46) into (34), we obtain the linear ODE

$$
\begin{aligned}
\left(\nu_{\max }\right. & \left.-\left(\nu_{\max }-\nu_{\min }\right) x\right) \frac{\mathrm{d}^{2} q_{U}}{\mathrm{~d} x^{2}} \\
& -\left(\nu_{\max }-\nu_{\min }\right) \frac{\mathrm{d} q_{U}}{\mathrm{~d} x}-\beta\left(q_{U}-q_{l}\right)=0 .
\end{aligned}
$$

It can be shown by substitution

$$
\begin{aligned}
& y^{2}=4 \delta_{*}\left(\nu_{*}-x\right), \quad q_{V}=q_{U}-q_{l}, \\
& \nu_{*}=\frac{\nu_{\max }}{\nu_{\max }-\nu_{\min }}>1, \\
& \delta_{*}=\frac{\beta}{\left(\nu_{\max }-\nu_{\min }\right)}>0
\end{aligned}
$$

that the linear ODE (Eqn. 477) reduces to the modified Bessel equation of the zero order (see, e.g., Polyanin and Zaitsev, 2003)

$$
y^{2} \frac{\mathrm{d}^{2} q_{V}}{\mathrm{~d} y^{2}}+y \frac{\mathrm{d} q_{V}}{\mathrm{~d} y}-y^{2} q_{V}=0 .
$$

The general solution of Eqn. 49] is well known. Hence, using the formulae (48), we obtain the solution of Eqn. (47):

$$
\begin{aligned}
q_{U}=C_{1} I_{0}\left(2 \sqrt{\delta_{*}\left(\nu_{*}-x\right)}\right) & \\
& +C_{2} K_{0}\left(2 \sqrt{\delta_{*}\left(\nu_{*}-x\right)}\right)+q_{l},
\end{aligned}
$$

where $I_{0}$ and $K_{0}$ are modified Bessel functions of the first and third kinds, respectively.

Substituting the obtained function $q_{U}$ into Eqn. (33) and using the well-known relations between the Bessel functions (Bateman, 1974), we find the function

$$
\begin{aligned}
j_{U}= & -L \sqrt{\frac{\nu_{*}-x}{\delta_{*}}}\left(C_{1} I_{1}\left(2 \sqrt{\delta_{*}\left(\nu_{*}-x\right)}\right)\right. \\
& \left.-C_{2} K_{1}\left(2 \sqrt{\delta_{*}\left(\nu_{*}-x\right)}\right)\right)
\end{aligned}
$$

where $I_{1}$ and $K_{1}$ are modified Bessel functions. Note that, similarly to the previous case, the constants $C_{1}$ and $C_{2}$ can be determined from the boundary conditions. Omitting rather simple calculations, we present only the result:

$$
\begin{aligned}
& C_{1}=\frac{\left(q_{0}-q_{l}\right) K_{1}\left(2 \sqrt{\delta_{*}\left(\nu_{*}-1\right)}\right)}{\Delta}, \\
& C_{2}=\frac{\left(q_{0}-q_{l}\right) I_{1}\left(2 \sqrt{\delta_{*}\left(\nu_{*}-1\right)}\right)}{\Delta},
\end{aligned}
$$

where

$$
\begin{aligned}
\Delta \equiv & I_{0}\left(2 \sqrt{\delta_{*} \nu_{*}}\right) K_{1}\left(2 \sqrt{\delta_{*}\left(\nu_{*}-1\right)}\right) \\
& +K_{0}\left(2 \sqrt{\delta_{*} \nu_{*}}\right) I_{1}\left(2 \sqrt{\delta_{*}\left(\nu_{*}-1\right)}\right)
\end{aligned}
$$

and $q_{0}$ is defined by (41).

Thus, we have found the explicit formulae for $q_{U}$ and $j_{U}$. Having the formulae (50)-51), the system of ODEs 35- 36 can be reduced to two linear autonomous ODEs with the unknown functions $u(x)$ and $w(x)$. These equations have the form

$$
\begin{gathered}
d_{1}\left(\nu_{\max }-\nu_{\min }\right)\left(\left(\nu_{*}-x\right) \frac{\mathrm{d}^{2} u}{\mathrm{~d} x^{2}}-\frac{\mathrm{d} u}{\mathrm{~d} x}\right) \\
+\frac{S_{G}}{\sqrt{\delta}_{*}} \frac{\mathrm{d}}{\mathrm{d} x}\left(\sqrt{\nu_{*}-x} \Delta_{1}(x) u\right) \\
+\left(S_{G} \Delta_{0}(x)-\kappa_{1}\right) u-u_{01}=0
\end{gathered}
$$

and

$$
\begin{aligned}
& d_{2}\left(\nu_{\max }-\nu_{\min }\right)\left(\left(\nu_{*}-x\right) \frac{\mathrm{d}^{2} w}{\mathrm{~d} x^{2}}-\frac{\mathrm{d} w}{\mathrm{~d} x}\right) \\
& +\frac{S_{A}}{\sqrt{\delta}_{*}} \frac{\mathrm{d}}{\mathrm{d} x}\left(\sqrt{\nu_{*}-x} \Delta_{1}(x)\left(w-w_{0}\right)\right) \\
& +\left(S_{A} \Delta_{0}(x)-\kappa_{2}\right)\left(w-w_{0}\right)-w_{01}=0
\end{aligned}
$$

where

$$
\begin{aligned}
\Delta_{1}(x) \equiv & C_{1} I_{1}\left(2 \sqrt{\delta_{*}\left(\nu_{*}-x\right)}\right) \\
& -C_{2} K_{1}\left(2 \sqrt{\delta_{*}\left(\nu_{*}-x\right)}\right)
\end{aligned}
$$

and

$$
\begin{aligned}
\Delta_{0}(x) \equiv & C_{1} I_{0}\left(2 \sqrt{\delta_{*}\left(\nu_{*}-x\right)}\right) \\
& +C_{2} K_{0}\left(2 \sqrt{\delta_{*}\left(\nu_{*}-x\right)}\right) .
\end{aligned}
$$

Although both the equations are linear second order ODEs with the same structure, we could not find their general solutions because of their awkwardness. Thus, we solve them numerically together with the boundary conditions (26) -27) using the Maple program package. In the next section, realistic values of the parameters for the formulae 50 -55 will be selected and applied in numerical simulations to calculate the non-uniform steady-state solutions. 


\section{Numerical results and their biomedical interpretation}

Here we present numerical results based on the formulae derived in Section 3. Our aim is to check whether they are applicable for describing the fluid-glucose-albumin transport in peritoneal dialysis. The parameter values in these formulae were derived from experimental and clinical data and have been applied in previous mathematical studies (Van Liew, 1968; Imholz et al., 1998; Zakaria et al., 1999; Flessner, 2001; Smit et al. 2004a; 2004b; Waniewski, 2001; Stachowska-Pietka et al., 2006; 2007; Cherniha et al., 2007; Waniewski et al., 2009 ). Most of the parameters, especially those for water and glucose, were derived from experimental data or obtained by fitting the distributed model to clinical data, and are discussed in detail in a recent paper of Stachowska-Pietka et al. (2012).

Some phenomena, such as vasodilatation and change of the parameters of interstitial transport with the change of tissue hydration, were not included in our model because our objective was the mathematical analysis of the model, so its structure had to remain not too complicated. Nevertheless, the model covers all basic transport phenomena and provides a good background for further modifications. However, only numerical studies are available for such extended models (see Smit et al., 2004a; 2004b; Stachowska-Pietka et al., 2006). Furthermore, those parameters without firmly established experimental values (i.e., Staverman reflection coefficients), were varied to check their impact on the results of modelling. The diffusivity of albumin in the interstitium is not well known, but it is much lower than interstitial diffusivity of glucose (see the works of Waniewski (2001) and Stachowska-Pietka et al. (2012) for more details). The values of parameters and absolute constants applied in numerical simulations are listed in Table 1.

Let us consider the first case of a constant fractional fluid void volume, i.e., with the restrictions (31) and (37). We remind the reader that the assumption that $F$ is a constant was applied in many studies and this implies that $\nu(x)$ is also a constant. It seems to be reasonable to set $\nu_{m}=\left(\nu_{\max }+\nu_{\min }\right) / 2=0.26$, i.e., we assume that the fractional fluid void volume at the steady-state stage of the peritoneal transport is an intermediate value between its maximum and minimum. In order to compare the numerical results obtained here with those for osmotic peritoneal transport obtained in earlier models, in which albumin transport was not considered, we neglect the oncotic pressure as a driving fluid force across the tissue, i.e., we set the Staverman reflection coefficients for albumin $\sigma_{T A}=\sigma_{A}=0$. This means that the fluid flux across the tissue, $j_{U}$, and the fluid flux from blood to the tissue, $q_{U}$ (see the formulae (4) and (5)), do not depend on the albumin concentrations. The Staverman reflection coefficients for glucose in the tissue and in the capillary wall are equal to $\sigma_{T G}=\sigma_{G}=0.001$. Hereafter, the values of other parameters and absolute constants are taken from Table 1.

Figure 1 presents the spatial distributions of the steady-state density of the fluid flux from blood to the tissue $q_{U}$ and the fluid flux across the tissue $j_{U}$, calculated using the formulae (38)-(41). The negative sign of $j_{U}$ indicates the net fluid flux occurs across the tissue towards the peritoneal cavity. Therefore, it corresponds to the water removal by ultrafiltration. The monotonically decreasing (with the distance from the peritoneal surface) function $q_{U}(x)$ and the monotonically increasing function $j_{U}(x)$ are in agreement with experimental data and previously obtained numerical results for the models that took into account only the glucose transport (Cherniha et al., 2007; Waniewski et al., 2007). It should be stressed that in those previous models albumin transport was not considered and the restrictions (31) and 37) were not used.

Using the value of the fluid flux $j_{U}$ at the point $x=0$, one may calculate the reverse water flow (i.e., out of the tissue to the cavity). The total fluid outflow from the tissue to the cavity (ultrafiltration), calculated assuming that the surface area of the contact between the dialysis fluid and the peritoneum is equal to $5 \cdot 10^{3} \mathrm{~cm}^{2}$ (this surface area measured in 10 patients on peritoneal dialysis was found to be within the range from 0.41 to $0.76 \mathrm{~m}^{2}$ (Chagnac et al., 2002)), is about $0.90 \mathrm{~mL} / \mathrm{min}$. Note that a similar value was obtained previously by Cherniha et al., (2007) using numerical simulations. Moreover, it comes from the formula (4) for $x=0$ that the ultrafiltration increases with growing $\sigma_{T G}$. For example, if one sets $\sigma_{T G}=0.01$ into Eqn. (4) then the total fluid outflow from the tissue to the cavity is $5.2 \mathrm{~mL} / \mathrm{min}$, which is very close to the value obtained by Cherniha et al. (2007) for the same parameters.

Figure 2 presents the spatial distributions of the glucose concentration in the tissue for $\sigma_{T G}=0.001$ and $\sigma_{T G}=0.01$ (see Fig. 2, the top panel). The interstitial glucose concentration $C_{G}$ decreases rapidly with the distance from the peritoneal surface to the constant steady-state value of $C_{G}^{*}$ (see the formula (15) in the deeper tissue layer independently of the $\sigma_{T G}$ values and is practically $C_{G}^{*}$ for $x>0.3$ (see Fig. 2, the bottom panel, where both curves coincide). Thus, the width of the tissue layer with the increased glucose concentration (that is around $0.3 \mathrm{~cm}$ ) does not depend on $\sigma_{T G}$. This remains in agreement with the previous results obtained by Cherniha et al. (2007).

We may conclude that, although the restriction in the form of the assumption (37) is rather artificial from physiological point of view, the analytical formulae derived in Section 3 lead to results which are similar to 
those obtained earlier with numerical simulations of pure glucose and water peritoneal transport (Cherniha et al., 2007), where this assumption was not used.

Let us now consider the second case, which is more realistic, i.e., hereafter the restrictions (31) and (46) apply.

Remark 1. In the case $\sigma_{T A}=0.0$ the results obtained via the formulae (50)-(53) and the ODEs (54)-55) practically coincide with those presented above (see Fig. 1).

Now we assume that the Staverman reflection coefficient for albumin is non-zero and equal to $\sigma_{A}=$ $\sigma_{T A}=0.5$, i.e., the maximum value of $\sigma_{T A}$ (see Table 1 ) is taken. In other words, we assume that the oncotic pressure plays an important role, contrary to the previous case. Here the fluid flux across the tissue $j_{U}$ and the fluid flux from blood to the tissue $q_{U}$ (see the formulae (4) and (5)) depend on the interstitial concentrations of glucose and albumin. We performed many calculations using the formulae (50) - 53) and ODEs (54) - 55) for a wide range of values of the parameter $\sigma_{T G}$, from very small (0.001) to large $(0.03)$ values. Of course, other parameters can also be varied; however, we restricted ourselves to this parameter because it is included in assumption 31.

The results obtained for $\sigma_{T G}=0.001, \sigma_{T G}=0.002$ and $\sigma_{T G}=0.01$ are presented in Figs. 3 and 4. It is quite interesting that the profiles for functions $q_{U}(x)$ and $j_{U}(x)$ shown in Fig. 3 are very similar to those in Fig. 1, although the relevant formulae are essentially different (the reader may compare (50)- 53) with (38)-(41) and $\sigma_{T A}=0.5$. Moreover, the form of these profiles are the same for a wide range of the values of $\sigma_{T G}$.

Using the value of the fluid flux $j_{U}$ at the point $x=0$, one may again calculate the ultrafiltration flow to the peritoneal cavity that can be obtained under the assumed restrictions on the model parameters. In the case $\sigma_{T G}=$ 0.01 the total fluid outflow from the tissue to the cavity is approximately equal to $4.8 \mathrm{~mL} / \mathrm{min}$, while it is very small $(0.06 \mathrm{~mL} / \mathrm{min})$ for $\sigma_{T G}=0.001$. To obtain values of the ultrafiltration that correspond to those measured during peritoneal dialysis, we need to set $\sigma_{T G} \geq 0.02$. For example, setting $\sigma_{T G}=0.02$ and $\sigma_{T G}=0.03$, we obtain the ultrafiltration rates of $10 \mathrm{~mL} / \mathrm{min}$ and $15 \mathrm{~mL} / \mathrm{min}$, which are close to those measured in clinical conditions for similar boundary concentration of glucose (Smit et al., 2004a; 2004b; Waniewski et al., 1996a; 2009; Stachowska et al., 2012). The initial values of ultrafiltration remain in agreement with those obtained by our group in clinical studies (Waniewski et al., 1996b; Waniewski, 2007). The initial rates of ultrafiltration for $3.86 \%$ glucose solution were found to be of $15 \mathrm{~mL} / \mathrm{min}$, which was much higher than those for 2.27 and $1.36 \%$ glucose solutions, 8 and $6 \mathrm{ml} / \mathrm{min}$, respectively (Waniewski et al. 1996a; 1996b). Similar values in the range of $14-18 \mathrm{~mL} / \mathrm{min}$ were measured during the initial minute and much lower values of $4-8 \mathrm{~mL} / \mathrm{min}$ at the end of a $4 \mathrm{~h}$ dwell study with $3.86 \%$ glucose solution (Smit et al. 2004a; 2004b).

Table 1. Parameters of the model used for numerical analysis of peritoneal transport.

\begin{tabular}{|c|c|}
\hline Parameter name & $\begin{array}{l}\text { Parameter symbol, } \\
\text { value and unit }\end{array}$ \\
\hline Minimal fractional void volume & $\nu_{\min }=0.17$ \\
\hline Maximal fractional void volume & $\nu_{\max }=0.35$ \\
\hline $\begin{array}{l}\text { Staverman reflection coefficient } \\
\text { for glucose }\end{array}$ & $0<\sigma_{T G} \leq 0.03$ \\
\hline $\begin{array}{l}\text { Sieving coefficient of glucose } \\
\text { in the tissue }\end{array}$ & $S_{T G}=1-\sigma_{T G}$ \\
\hline $\begin{array}{l}\text { Staverman reflection coefficient } \\
\text { for albumin }\end{array}$ & $0.05 \leq \sigma_{T A} \leq 0.5$ \\
\hline $\begin{array}{l}\text { Sieving coefficient of albumin } \\
\text { in the tissue }\end{array}$ & $S_{T A}=1-\sigma_{T A}$ \\
\hline Hydraulic permeability of the tissue & $\begin{array}{l}K=5.14 \cdot 10^{-5} \\
\mathrm{~cm}^{2} / \mathrm{min} / \mathrm{mm} \mathrm{Hg}\end{array}$ \\
\hline Gas constant times temperature & $\begin{array}{l}R T=18 \cdot 10^{3} \\
\mathrm{~mm} \mathrm{Hg} \cdot \mathrm{mL} / \mathrm{mmol}\end{array}$ \\
\hline Width of the tissue layer & $L=1.0 \mathrm{~cm}$ \\
\hline $\begin{array}{l}\text { Hydraulic permeability of } \\
\text { capillary wall times density } \\
\text { of capillary surface area }\end{array}$ & $\begin{array}{l}L_{P} a=7.3 \cdot 10^{-5} \\
1 / \min / \mathrm{mm} \mathrm{Hg}\end{array}$ \\
\hline $\begin{array}{l}\text { Volumetric fluid flux to } \\
\text { lymphatic vessels }\end{array}$ & $\begin{array}{l}q_{l}=0.26 \cdot 10^{-4} \\
\min ^{-1}\end{array}$ \\
\hline $\begin{array}{l}\text { Diffusivity of glucose in the tissue } \\
\text { divided by } \nu_{\min }\end{array}$ & $\begin{array}{l}D_{G}=12.11 \cdot 10^{-5} \\
\mathrm{~cm}^{2} / \min \end{array}$ \\
\hline $\begin{array}{l}\text { Diffusivity of albumin in the tissue } \\
\text { divided by } \nu_{\min }\end{array}$ & $\begin{array}{l}D_{A}=0.2 \cdot 10^{-5} \\
\mathrm{~cm}^{2} / \min \end{array}$ \\
\hline $\begin{array}{l}\text { Permeability of the capillary wall } \\
\text { for glucose times density } \\
\text { of capillary surface area }\end{array}$ & $\begin{array}{l}p_{G} a= \\
3.4 \cdot 10^{-2} \min ^{-1}\end{array}$ \\
\hline $\begin{array}{l}\text { Permeability of the capillary wall } \\
\text { for albumin times density } \\
\text { of the capillary surface area }\end{array}$ & $\begin{array}{l}p_{A} a= \\
6 \cdot 10^{-5} \min ^{-1}\end{array}$ \\
\hline Glucose concentration in blood & $\begin{array}{l}C_{G B}=6 \cdot 10^{-3} \\
\mathrm{mmol} / \mathrm{mL}\end{array}$ \\
\hline Albumin concentration in blood & $\begin{array}{l}C_{A B}=0.6 \cdot 10^{-3} \\
\mathrm{mmol} / \mathrm{mL}\end{array}$ \\
\hline Glucose concentration in dialysate & $\begin{array}{l}C_{G D}=180 \cdot 10^{-3} \\
\mathrm{mmol} / \mathrm{mL}\end{array}$ \\
\hline Albumin concentration in dialysate & $C_{A D}=0$ \\
\hline Hydrostatic pressure of blood & $P_{B}=15 \mathrm{~mm} \mathrm{Hg}$ \\
\hline Hydrostatic pressure of dialysate & $P_{D}=12 \mathrm{~mm} \mathrm{Hg}$ \\
\hline Non-dimensional parameter & $\alpha=0.8$ \\
\hline
\end{tabular}

The spatial distributions of the glucose and albumin concentrations for different values of $\sigma_{T G}$ are displayed in Fig. 4. Note that the glucose concentration in the tissue, $C_{G}$, again decreases rapidly with the distance from the peritoneal cavity to the constant steady-state $C_{G}^{*}$ in the deeper tissue layer. The glucose concentration is practically equal to $C_{G}^{*}$ for any $x>0.1 \mathrm{~cm}$ if $\sigma_{T G}$ is 


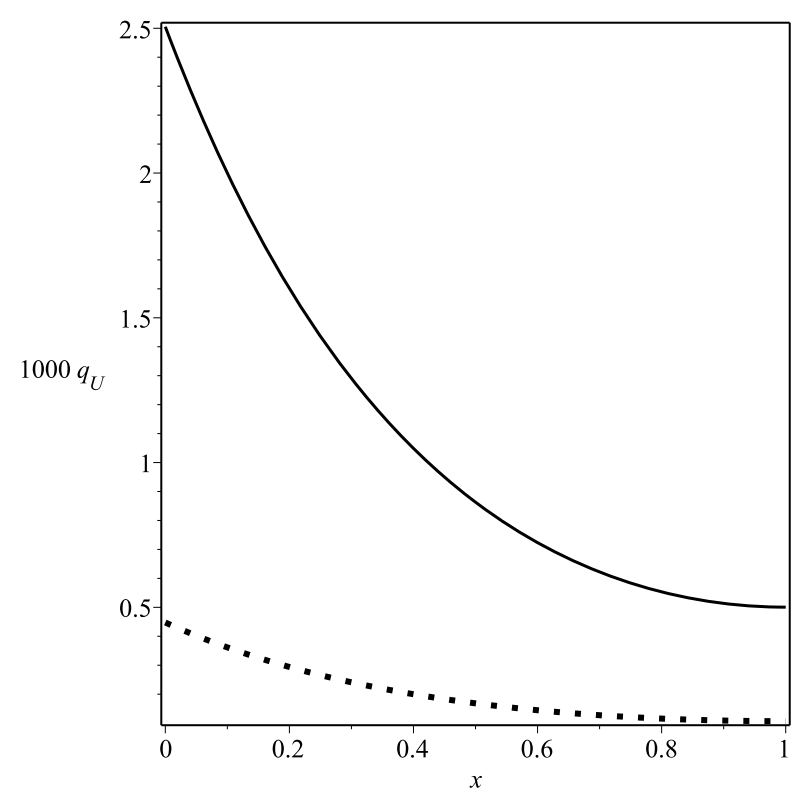

Fig. 1. Fluid fluxes from blood to tissue $q_{U}$ (in $\min ^{-1}$ ) and across the tissue $j_{U}\left(\right.$ in $\min ^{-1} \cdot \mathrm{cm}$ ) as a function of distance from peritoneal cavity $x$ (in $\mathrm{cm})$ for $\nu=\left(\nu_{\max }+\right.$ $\left.\nu_{\min }\right) / 2, \sigma_{T G}=0.001$ (dot curves), $\sigma_{T G}=0.01$ (solid curves), and $\sigma_{T A}=0.0$.

large ( $\geq 0.01$ ). The tissue layer with non-constant $C_{G}$ is slightly wider if $\sigma_{T G}$ is small $(\leq 0.002)$. For such values of $\sigma_{T G}$, the glucose concentration is equal to $C_{G}^{*}$ for all $x>0.2 \mathrm{~cm}$.

The albumin concentration in the tissue, $C_{A}$, is decreased (in the direction to the peritoneal cavity) in a thin layer, whereas it remains unperturbed in the deeper tissue layers (Fig. 4). This decrease corresponds to the transport of albumin to the peritoneal cavity, and is most pronounced close to the peritoneal surface. We found that the albumin concentration essentially depends on the parameter $\sigma_{T G}$. In fact, three curves presented in Fig. 4 show that the tissue layer with decreased $C_{A}$ is wide for $\sigma_{T G}=0.001$, whereas it is much smaller for $\sigma_{T G}=0.002$ and almost vanishes for $\sigma_{T G}=0.01$. In the case $\sigma_{T G}=0.001$, the tissue layer of decreased $C_{A}$ is about $0.3 \mathrm{~cm}$, indicating the removal of albumin from this part of the tissue. In the case of high $\sigma_{T G}$, the albumin concentration in the tissue is decreased only in a very thin layer, while it remains at a physiological level and equal to $C_{A}^{*}$ (see the formula (15)) beyond this layer. Thus, the high ultrafiltration flow contributes to fast inflow of albumin from blood to the tissue and drags albumin from deep to subsurface layers. However, the diffusive leak of albumin from the tissue to the peritoneal cavity is faster with high ultrafiltration because of a higher concentration gradient (Fig. 4).
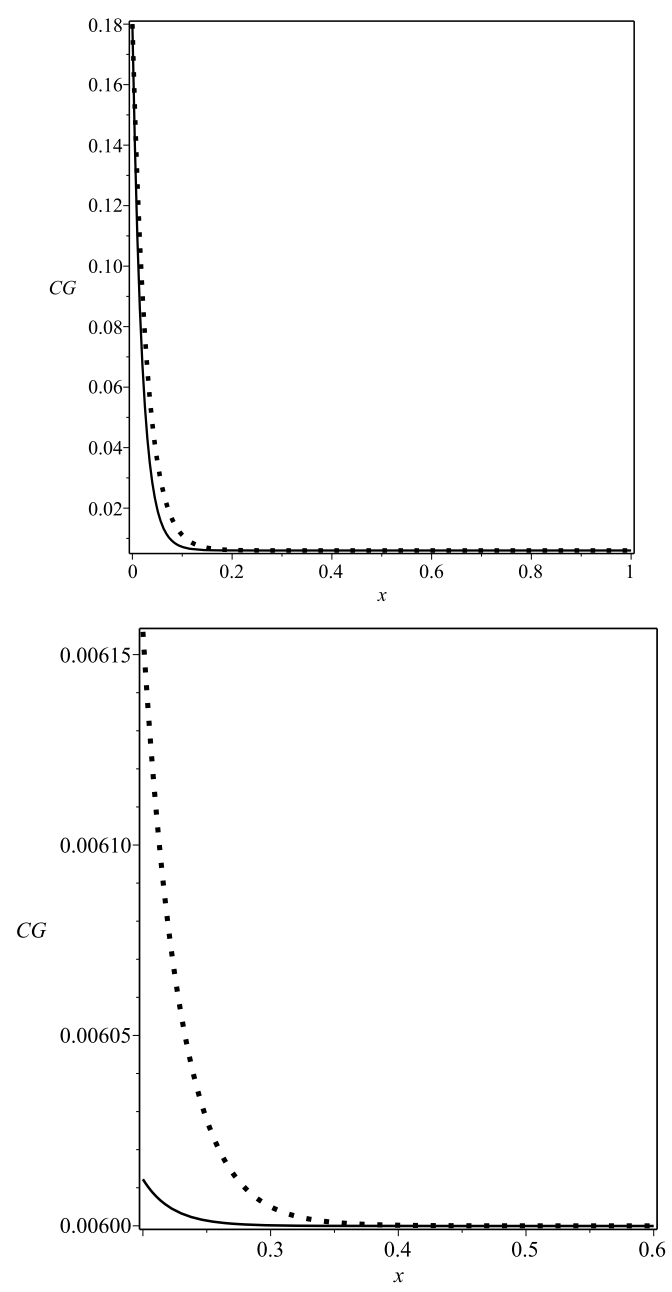

Fig. 2. Profiles of glucose concentration in the tissue $C_{G}$ (in $\left.\mathrm{mmol} \cdot \mathrm{mL}^{-1}\right)$ as a function of distance from the peritoneal cavity $x$ (in $\mathrm{cm}$ ) for $\nu=\left(\nu_{\max }+\nu_{\min }\right) / 2$, $\sigma_{T G}=0.001$ (dot curves) and $\sigma_{T G}=0.01$ (solid curves). Upper panel for $0 \leq x \leq 1$, bottom panel: zoom for $0.2 \leq x \leq 0.6$.

\section{Conclusions}

In this paper, a new mathematical model for fluid transport in peritoneal dialysis was constructed. The model is based on a three-component nonlinear system of two-dimensional partial differential equations and the relevant boundary and initial conditions. To analyze the non-uniform steady-state solutions, the model was reduced to a non-dimensional form. Under additional assumptions the problem was simplified in order to obtain analytical solutions in an explicit form. As a result, the exact formulae for the density of the fluid flux from blood to the tissue and the fluid flux across the tissue were constructed, together with two linear autonomous ODEs for glucose and albumin concentrations in the tissue.

The analytical results were checked for their applicability to describe the fluid-glucose-albumin 

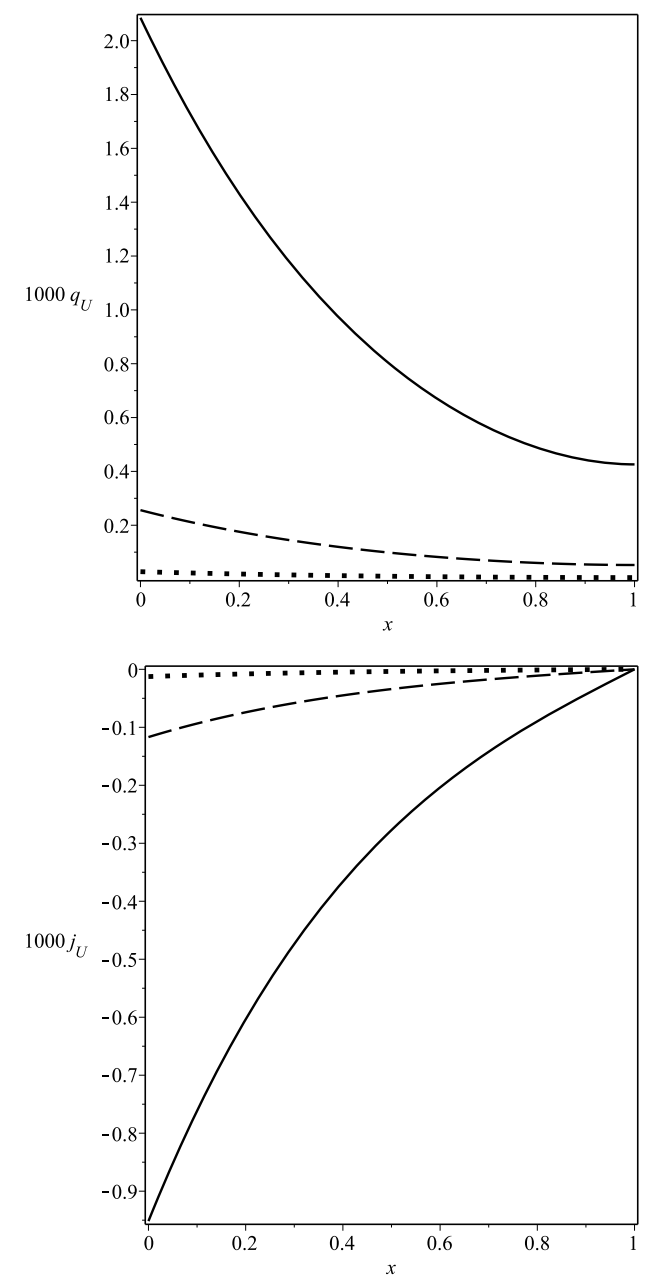

Fig. 3. Fluid flux from blood to the tissue $q_{U}\left(\right.$ in $\left.\min ^{-1}\right)$ and the fluid flux across the tissue $j_{U}\left(\right.$ in $\left.\min ^{-1} \cdot \mathrm{cm}\right)$ as a function of distance from the peritoneal cavity $x$ (in $\mathrm{cm})$ for $\nu=\nu_{\max }-\left(\nu_{\max }-\nu_{\min }\right) x, \sigma_{T A}=0.5$, and $\sigma_{T G}=0.001$ (dot curves), 0.002 (dash curves), 0.01 (solid curves).

transport in peritoneal dialysis. The selected values of the parameters were based on previous experimental and clinical studies or estimated from the data using the distributed model. Some of the parameters (Staverman reflection coefficients) were varied to check their impact on the model predictions. The model presented in the current study was extended, compared with previous models, by including the transport of water and the two most important solutes related to water transport: glucose, which is used as an osmotic agent, and albumin, which is the primary determinant of oncotic pressure.

These two solutes differ greatly in molecular mass (by a factor of 300) and therefore also differ in their transport parameters. The other studies include mostly only one of these two solutes into the model (Flessner $e t$ al., 1984; Baxter and Jain, 1989; 1990; 1991; Cherniha and Waniewski 2005; Flessner 2006; Cherniha et al.,
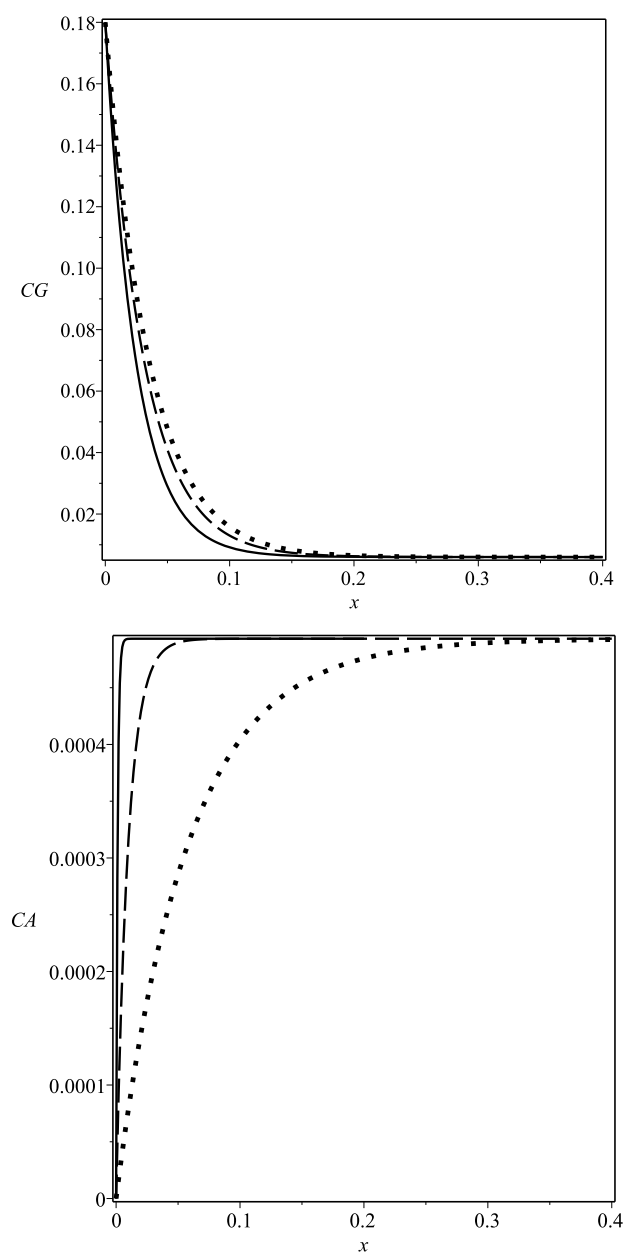

Fig. 4. Glucose concentration profiles, $C_{G}$ (in $\mathrm{mmol} \cdot \mathrm{mL}^{-1}$ ), and albumin concentration profiles, $C_{A}$ (in mmol . $\mathrm{mL}^{-1}$ ), in the tissue as a function of distance from the peritoneal cavity, $x$ (in $\mathrm{cm}$ ), for $\nu=\nu_{\max }-\left(\nu_{\max }-\right.$ $\left.\nu_{\min }\right) x, \sigma_{T A}=0.5$, and $\sigma_{T G}=0.001$ (dot curves), 0.002 (dash curves), 0.01 (solid curves).

2007; Waniewski et al., 2007; 2009; Stachowska-Pietka et al., 2007; 2012). On the other hand, our investigations are restricted to the steady state solutions, whereas in real dialysis the fluid and solute transport changes because of the change in boundary conditions (Stachowska-Pietka et $a l ., 2006)$. We did not include in the model the phenomena of vasodilation and change in tissue hydration that yield spatially non-uniform structure of the tissue (however, our $x$-dependent fractional volume of the interstitial fluid $\nu$ partially accounts for this non-uniform structure) and contribute to the details of numerical solutions as compared to clinical data (see Smit et al., 2004a). Only some of the model predictions can be compared directly with clinical data. The most important output is the rate of ultrafiltration of water to the peritoneal cavity that is induced by glucose. With the concentration of glucose applied in our calculations, the ultrafiltration rate 
of about $15 \mathrm{~mL} / \mathrm{min}$ is expected (Heimbürger et al., 1992; Waniewski et al., 1996a; 1996b; Smit et al 2004a; 2004b).

Our results demonstrate that this value can be obtained if the reflection coefficient for glucose is high (0.02-0.03). This general observation is in agreement with early measurements of this coefficient, but differs from much higher values of the coefficient estimated by previous numerical simulations (Stachowska-Pietka et al., 2006; Waniewski, 2007; Waniewski et al., 2007). The difference might be explained by that between glucose reflection coefficients for the tissue (low) and the capillary wall (high) obtained from previous numerical simulations, whereas these two coefficients were equal (with a medium value to yield the demanded ultrafiltration rate, for the sake of mathematical tractability) in our predictions (see below).

The glucose and albumin profiles obtained from our model are similar to those found in experimental studies (although no such data are available for humans, they are for rats) and to the previous numerical simulations of clinical dialysis (Flessner et al., 2006; Waniewski et al., 2009; Stachowska-Pietka et al, 2012). The glucose interstitial concentration sharply decreases within $2 \mathrm{~mm}$ from the peritoneal surface and is equal to its blood concentration in deeper tissue layers (see Figs. 2 and 4) as found in other numerical studies (Waniewski et al. 2009; Stachowska-Pietka et al., 2012). A similar profile was found in experiments performed in rats with manitol, which has identical transport characteristics to glucose (Flessner et al., 2006). In contrast, the interstitial concentration of albumin is high within the deep layers of the tissue and decreases sharply in a thin layer close to the peritoneal tissue, as shown in Figs. 2 and 4. This low subperitoneal protein concentration (and therefore low oncotic pressure) was confirmed experimentally (Rosengren et al., 2004) and in numerical simulations (Stachowska-Pietka et al., 2007). Other results, such as the profiles of the flux from blood to the tissue $q_{U}$ and the flux across the tissue, $j_{U}$ (see Figs. 1 and 3), do not have experimental counterparts and are rarely presented as results of numerical studies.

Thus, our model, although aimed at the investigation of its mathematical structure with specific coefficient conditions, also yielded interesting predictions, in spite of the simple approximations for the fractional interstitial fluid volume $\nu$ that were applied. Even the simplest approximations of $\nu$ by a constant or a linear function yielded predictions in agreement with the models based on nonlinear dependence of $\nu$ on interstitial hydrostatic pressure. In fact, the monotonically decreasing (with the distance from the peritoneal surface) function $q_{U}(x)$, describing the fluid flux from blood to the tissue, and the monotonically increasing function $j_{U}(x)$, describing the fluid flux across the tissue, are in agreement with experimental data and previously obtained numerical results. Moreover, we calculated the fluid flux $j_{U}(t, x)$ at $x=0$, which describes the net ultrafiltration flow, i.e., the efficiency of removal of water during peritoneal dialysis, because it is important from a practical point of view. The results show that the Staverman reflection coefficient for glucose $\sigma_{T G}$ plays a crucial role for ultrafiltration. To obtain the values of the ultrafiltration corresponding to experimental data, $10-20 \mathrm{~mL} / \mathrm{min}$, measured during peritoneal dialysis, we need to set $\sigma_{T G} \geq 0.02$ in the formulae obtained.

The finding that high ultrafiltration flow rates measured in clinical studies may be obtained with a relatively low $\sigma_{G}$ of $0.01-0.03$ and at the same time rather high $\sigma_{T G}=\sigma_{G}$ (which is the assumption necessary to get the presented above analytical solutions) is interesting. In fact, much higher values of $\sigma_{G}$ (about 0.5 ) and lower values of $\sigma_{T G}$ (about 0.005 ) were used by (Waniewski et al., 2009; Stachowska-Pietka et al., 2012) to obtain similar flow rates. Our solutions constructed above add a new perspective to the unsolved problem of the values of $\sigma_{G}$ (see the detailed discussion by Waniewski et al. (2009) and Stachowska-Pietka et al. (2012)). Thus, these new results should be further pursued not only because of mathematical interest but also of their potential practical applications.

The differences between the present analytical solutions and the previous simulations also include the profile of the fluid void volume (approximated due to the linear function), which is an input to our model equations, and not an outcome of the simulations. Other approximations of the fractional fluid volume $\nu$ may in future result in similar exact formulae. In the particular case, the preliminary calculations show that such exact formulae can be obtained when $\nu$ is a decreasing exponential function. However, the assumption about the equality of the reflection coefficients in the tissue and in the capillary wall, which demonstrates an interesting specific symmetry in the equations, can be too restrictive for practical applications of the derived formulae (Waniewski et al., 2009). Therefore, other approaches to find the analytical solutions of the model need to be looked for.

\section{Acknowledgment}

This work was conducted within the project Mathematical models of fluid and solute transport in normal and pathological tissue supported by the Mianowski Fund (Warsaw, Poland) and the joint project Mathematical modeling transport processes in tissue during peritoneal dialysis between the PAS and the NAS of Ukraine.

\section{References}

Bateman, H. (1985). Higher Transcendental Functions, Vol. 2. Robert E. Krieger Publishing Company, Malabar, FL. 
Baxter, L.T. and Jain, R.K. (1989). Transport of fluid and macromolecules in tumors, I: Role of interstitial pressure and convection, Microvascular Research 37(1): 77-104.

Baxter, L.T. and Jain, R.K. (1990). Transport of fluid and macromolecules in tumors, II: Role of heterogeneous perfusion and lymphatics, Microvascular Research 40(2): 246-263.

Baxter, L.T. and Jain, R.K. (1991). Transport of fluid and macromolecules in tumors, III: Role of binding and metabolism, Microvascular Research 41(1): 5-23.

Chagnac, A., Herskovitz, P., Ori Y., Weinstein, T., Hirsh, J., Katz, M. and Gafter, U. (2002). Effect of increased dialysate volume on peritoneal surface area among peritoneal dialysis patients, Journal of the American Society of Nephrology 13(10): 2554-2559.

Cherniha, R., Dutka, V., Stachowska-Pietka, J. and Waniewski, J. (2007). Fluid transport in peritoneal dialysis: A mathematical model and numerical solutions, in $\mathrm{A}$. Deutsch, L. Brusch, H. Byrne, G. de Vries and H.P. Herzel (Eds.), Mathematical Modeling of Biological Systems, Vol. I, Birkhaeuser, Boston, MA, pp. 291-298.

Cherniha, R. and Waniewski, J. (2005). Exact solutions of a mathematical model for fluid transport in peritoneal dialysis, Ukrainian Mathematical Journal 57(8): 1112-1119.

Collins, J.M. (1981). Inert gas exchange of subcutaneous and intraperitoneal gas pockets in piglets, Respiration Physio$\operatorname{logy}$ 46(3): 391-404.

Czyzewska, K., Szary, B. and Waniewski, J. (2000). Transperitoneal transport of glucose in vitro, Artificial Organs 24(11): 857-863.

Dedrick, R.L., Flessner, M.F., Collins, J.M. and Schultz, J.S. (1982). Is the peritoneum a membrane? Journal of American Society for Artificial Internal Organs 5(1): 1-8.

Flessner, M.F. (1994). Osmotic barrier of the parietal peritoneum, American Journal of Physiology 267(5): F861-870.

Flessner, M.F. (2001). Transport of protein in the abdominal wall during intraperitoneal therapy, I: Theoretical approach, American Journal of Physiology-Gastrointestinal and Liver Physiology 281(2): G424-437.

Flessner, M.F. (2006). Peritoneal ultrafiltration: Mechanisms and measures, Contributions to Nephrology 150: 28-36.

Flessner, M.F. (2009). Peritoneal ultrafiltration: Physiology and failure, Contributions to Nephrology 163: 7-14.

Flessner, M.F., Deverkadra, R., Smitherman, J., Li, X. and Credit, K. (2006). In vivo determination of diffusive transport parameters in a superfused tissue, American Journal of Physiology—Renal Physiology 291(5): F1096-1103.

Flessner, M.F., Dedrick, R.L. and Schultz J.S. (1984). A distributed model of peritoneal-plasma transport: Theoretical considerations, American Journal of Physiology 246(4): R597-607.
Flessner, M. F., Fenstermacher, J.D., Dedrick, R.L. and Blasberg, R.G. (1985). A distributed model of peritoneal-plasma transport: Tissue concentration gradients, American Journal of Physiology 248(3): F425-435.

Gokal, R. and Nolph, K.D. (1994). The Textbook of Peritoneal Dialysis, Kluwer, Dordrecht.

Guest, S., Akonur, A., Ghaffari, A., Sloand, J. and Leypoldt, J. K. (2012). Intermittent peritoneal dialysis: Urea kinetic modeling and implication of residual kidney function, $\mathrm{Pe}$ ritoneal Dialysis International 32(2): 142-148.

Gupta, E., Wientjes, M.G. and Au, J.L. (1995). Penetration kinetics of 2', 3'-dideoxyinosine in dermis is described by the distributed model, Pharmaceutical Research 12(1): 108-112.

Heimbürger, O., Waniewski, J., Werynski, A. and Lindholm, B. (1992). A quantitative description of solute and fluid transport during peritoneal dialysis, Kidney International 41(5): 1320-1332.

Imholz, A.L., Koomen, G.C., Voorn, W.J., Struijk, D.G., Arisz, L. and Krediet, R.T. (1998). Day-to-day variability of fluid and solute transport in upright and recumbent positions during CAPD, Nephrology Dialysis Transplantation 13(1): 146-153.

Katchalsky, A. and Curran, P.F. (1965). Nonequilibrium Thermodynamics in Biophysics, Harvard University Press, Cambridge.

Landis, E.M. and Pappenheimer, J.R. (1963). Exchange of Substances Through the Capillary Walls. Handbook of Physiology. Circulation, American Physiological Society, Washington, DC.

Parikova, A., Smit, W., Struijk, D.G. and Krediet, R.T. (2006). Analysis of fluid transport pathways and their determinants in peritoneal dialysis patients with ultrafiltration failure, Kidney International 70(11): 1988-1994.

Patlak, C.S. and Fenstermacher, J.D. (1975). Measurements of dog blood-brain transfer constants by ventriculocisternal perfusion, American Journal of Physiology 229(4): 877-884.

Perl, W. (1962). Heat and matter distribution in body tissues and the determination of tissue blood flow by local clearance methods, Journal of Theoretical Biology 2(3): 201-235.

Perl, W. (1963). An extension of the diffusion equation to include clearance by capillary blood flow, Annals of the New York Academy of Sciences 108: 92-105.

Piiper, J., Canfield, R.E. and Rahn, H. (1962) Absorption of various inert gases from subcutaneous gas pockets in rats, Journal of Applied Physiology 17(2): 268-274.

Polyanin, A.D. and Zaitsev, V.F. (2003). Handbook of Exact Solutions for Ordinary Differential Equations, CRC Press Company, Boca Raton, FL.

Rosengren, B.I., Carlsson, O., Venturoli, D., al Rayyes, O. and Rippe, B. (2004). Transvascular passage of macromolecules into the peritoneal cavity of normo- and hypothermic rats in vivo: Active or passive transport? $\mathrm{Jo}$ urnal of Vascular Research 41(2): 123-130. 
Seames, E.L., Moncrief, J.W. and Popovich, R.P. (1990). A distributed model of fluid and mass transfer in peritoneal dialysis. American Journal of Physiology 258(4): R958-972.

Smit, W., Struijk, D.G., Pannekeet, M.M. and Krediet, R.T. (2004a). Quantification of free water transport in peritoneal dialysis, Kidney International 66(2): 849-854.

Smit, W., van Esch, S., Struijk, D.G., Pannekeet, M.M. and Krediet, R.T. (2004b). Free water transport in patients starting with peritoneal dialysis: A comparison between diabetic and non diabetic patients, Advances in Peritoneal Dialysis 20: 13-17.

Stachowska-Pietka, J., Waniewski, J., Flessner, M.F. and Lindholm, B. (2006). Distributed model of peritoneal fluid absorption, American Journal of Physiology-Heart and Circulatory Physiology 291(4): H1862-1874.

Stachowska-Pietka, J., Waniewski, J., Flessner, M.F. and Lindholm, B. (2007). A distributed model of bidirectional protein transport during peritoneal fluid absorption, Advances in Peritoneal Dialysis 23: 23-27.

Stachowska-Pietka, J., Waniewski, J., Flessner, M.F. and Lindholm, B. (2012). Computer simulations of osmotic ultrafiltration and small solute transport in peritoneal dialysis: A spatially distributed approach, American Journal of Physiology-Renal Physiology 302(10): F1331-1341.

Van Liew, H.D. (1968). Coupling of diffusion and perfusion in gas exit from subcutaneous pocket in rats, American Journal of Physiology 214(5): 1176-1185.

Waniewski, J. (2001). Physiological interpretation of solute transport parameters for peritoneal dialysis, Computational and Mathematical Networks in Medicine 3(3): 177-190.

Waniewski, J. (2002). Distributed modeling of diffusive solute transport in peritoneal dialysis, Annals of Biomedical Engineering 30(9): 1181-1195.

Waniewski, J. (2007). Mean transit time and mean residence time for linear diffusion-convection-reaction transport system, Computational and Mathematical Methods in Medicine 8(1): 37-49.

Waniewski, J.(2008). Transit time, residence time, and the rate of approach to steady state for solute transport during peritoneal dialysis, Annals of Biomedical Engineering 36: $1735-1743$.

Waniewski, J.(2013). Peritoneal fluid transport: Mechanisms, pathways, methods of assessment, Archives of Medical Research 44(8): 576-583.

Waniewski, J., Dutka, V., Stachowska-Pietka, J. and Cherniha, R. (2007). Distributed modeling of glucose-induced osmotic flow, Advances in Peritoneal Dialysis 23: 2-6.

Waniewski, J., Heimbürger, O., Werynski, A. and Lindholm, B. (1996a). Simple models for fluid transport during peritoneal dialysis, International Journal of Artificial Organs 19(8): 455-466.

Waniewski, J., Heimbürger, O., Werynski, A. and Lindholm, B. (1996b). Osmotic conductance of the peritoneum in CAPD patients with permanent loss of ultrafiltration capacity, $P e$ ritoneal Dialysis International 16(5): 488-496.
Waniewski, J., Stachowska-Pietka, J. and Flessner, M.F. (2009). Distributed modeling of osmotically driven fluid transport in peritoneal dialysis: Theoretical and computational investigations, American Journal of Physiology-Heart and Circulatory Physiology 296(6): H1960-1968.

Wientjes, M.G., Badalament, R.A., Wang, R.C., Hassan, F. and $\mathrm{Au}$, J.L. (1993). Penetration of mitomycin C in human bladder, Cancer Research 53(14): 3314-3320.

Wientjes, M.G., Dalton, J.T., Badalament, R.A., Drago, J.R. and Au, J.L. (1991). Bladder wall penetration of intravesical mitomycin $\mathrm{C}$ in dogs, Cancer Research 51(16): 4347-4354.

Zakaria, E.R., Lofthouse, J. and Flessner, M.F. (1999). In vivo effects of hydrostatic pressure on interstitium of abdominal wall muscle, American Journal of Physiology 276(2): H517-529.

Zakaria, E.R., Lofthouse, J. and Flessner, M.F. (2000). Effect of intraperitoneal pressures on tissue water of the abdominal muscle, American Journal of Physiology—Renal Physiology 278(6): F875-885.

Roman Cherniha received his M.Sc. degree in mathematics from the Faculty of Mechanics and Mathematics, Kyiv Taras Shevchenko State University, in 1981. He obtained a Ph.D. degree in differential equations in 1987 from the Institute of Mathematics, National Academy of Sciences of Ukraine, and a D.Sc. degree in mathematical physics in 2003 from the same institute. The professorial title (mathematics) was granted to him by the government of Ukraine in 2012. His fields of research include application of modern methods (including Lie and conditional symmetry based approaches) for analytical solving nonlinear boundary value problems arising in mathematical physics and mathematical biology.

Joanna Stachowska-Piętka received her M.Sc. degree in mathematics from the Faculty of Mathematics, Informatics and Mechanics, University of Warsaw, in 2000, and in economics from the Faculty of Economic Sciences, University of Warsaw, in 2002. She obtained a Ph.D. degree in biomedical engineering from the Institute of Biocybernetics and Biomedical Engineering of the Polish Academy of Sciences in 2011. Her research is focused on local fluid balance in the human body, fluid and solute transport through the tissue and across the blood capillary wall. Her topics of current research are related to fluid and solute transport in patients on dialysis.

Jacek Waniewski received his M.Sc. degree in physics from the Faculty of Physics, Warsaw University, in 1973. He obtained a Ph.D. degree in physics in 1983 from the same faculty and in medicine in 2001 from Karolinska Institutet, Stockholm. His D.Sc. degree in biomedical engineering was granted to him by the Institute of Biocybernetics and Biomedical Engineering of the Polish Academy of Sciences in 1995. He obtained the professorial title (technical sciences) in 2012. His research is currently focused on the applications of mathematical modelling in medicine and biomedical research with emphasis on clinical problems related to patients with kidney failure.

Received: 7 October 2013 Revised: 6 May 2014 